\title{
LA INSCRIPCIÓN EN CARACTERES IBÉRICOS DE LOS MAÍLLOS (BELVÍS DE LA JARA, TOLEDO) *
}

\author{
POR \\ EUGENIO RAMÓN LUJÁN MARTÍNEZ \\ Universidad Complutense de Madrid
}

\section{RESUMEN}

Se estudia una inscripción en caracteres ibéricos aparecida en el término municipal de Belvís de la Jara (Toledo). Su gran interés radica en el lugar de hallazgo, que hace variar significativamente hacia Occidente los límites de distribución del semisilabario levantino.

\section{SUMMARY}

We study in this paper an inscription written in Iberian script found in Belvís de la Jara (province of Toledo, central Spain). Its importance lies in the fact that no other inscription of this kind has been found so far this West of the territory usually ascribed to the Levantine semisillabary.

\section{INTRODUCCIÓN}

La inscripción ${ }^{1}$ que vamos a estudiar apareció fragmentada en dos piezas en una pared de piedra del camino que va desde las casas de labranza de Los Maíllos (término municipal de Belvís de la Jara, provincia de Toledo) a unos pequeños huertos de la vegata del arroyo del mismo nombre (fig. 1). Los frag-

* Quiero expresar mi agradecimiento al Prof. Fernando Jiménez de Gregorio, que me ha dado todas las facilidades para el estudio de la inscripción. También estoy muy agradecido al Prof. Javier de $\mathrm{Hoz}$, que tuvo la amabilidad de leer una versión anterior de este trabajo y me hizo interesantes observaciones que han contribuido a mejorarlo decisivamente. Por supuesto la responsabilidad de los errores que pueda contener es sólo mía.

1 Fue dada a conocer el 11 de noviembre de 1981 por el Prof. Jiménez de Gregorio en La Voz del Tajo; sobre ella volvió a llamar la atención en el mismo medio de comunicación el 1 de octubre de 1983 y, posteriormente, la recogió en un artículo del año 1989 junto con otros hallazgos arqueológicos realizados en la misma provincia, así como en su trabajo aparecido en 1992. Con todo -y a pesar de que la inscripción es de gran interés en el marco de la epigrafía de las lenguas prerromanas de la península Ibérica- hasta el momento no se ha llevado a cabo la edición y un estudio de detalle de la misma.

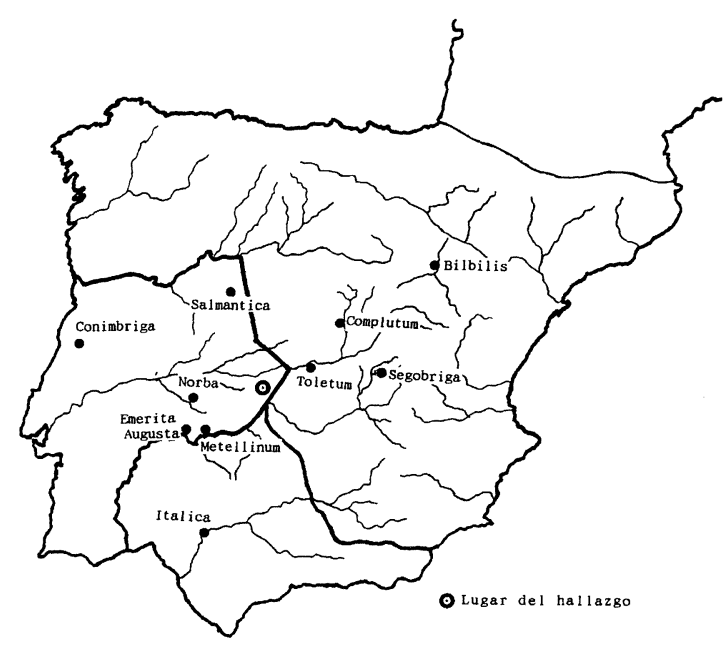

Fig. 1.-Lugar de hallazgo de la inscripción.

mentos aparecieron separados uno del otro por unos $11 \mathrm{~m}$ y se puede pensar que la piedra — una cuarcita amarillenta - fue partida precisamente para su utilización en dicha pared ${ }^{2}$. Que ambos formaban originariamente una unidad resulta indiscutible, ya que encajan perfectamente. Las medidas del soporte en su estado actual son de $87,7 \mathrm{~cm}$ de largo en la parte central y de $51,5 \mathrm{~cm}$ de altura. Vid. fig. 2 .

\section{ESTUDIO DE LA INSCRIPCIÓN}

\subsection{Observaciones generales}

El sistema gráfico empleado en la inscripción ha sido el semisilabario levantino, por lo que su importancia radica principalmente en el lugar de aparición, que hace variar significativamente hacia Occidente los límites de distribución de esta variedad de las es-

${ }^{2}$ Para las circunstancias del hallazgo vid. Jiménez de Gregorio (1989: 31). 


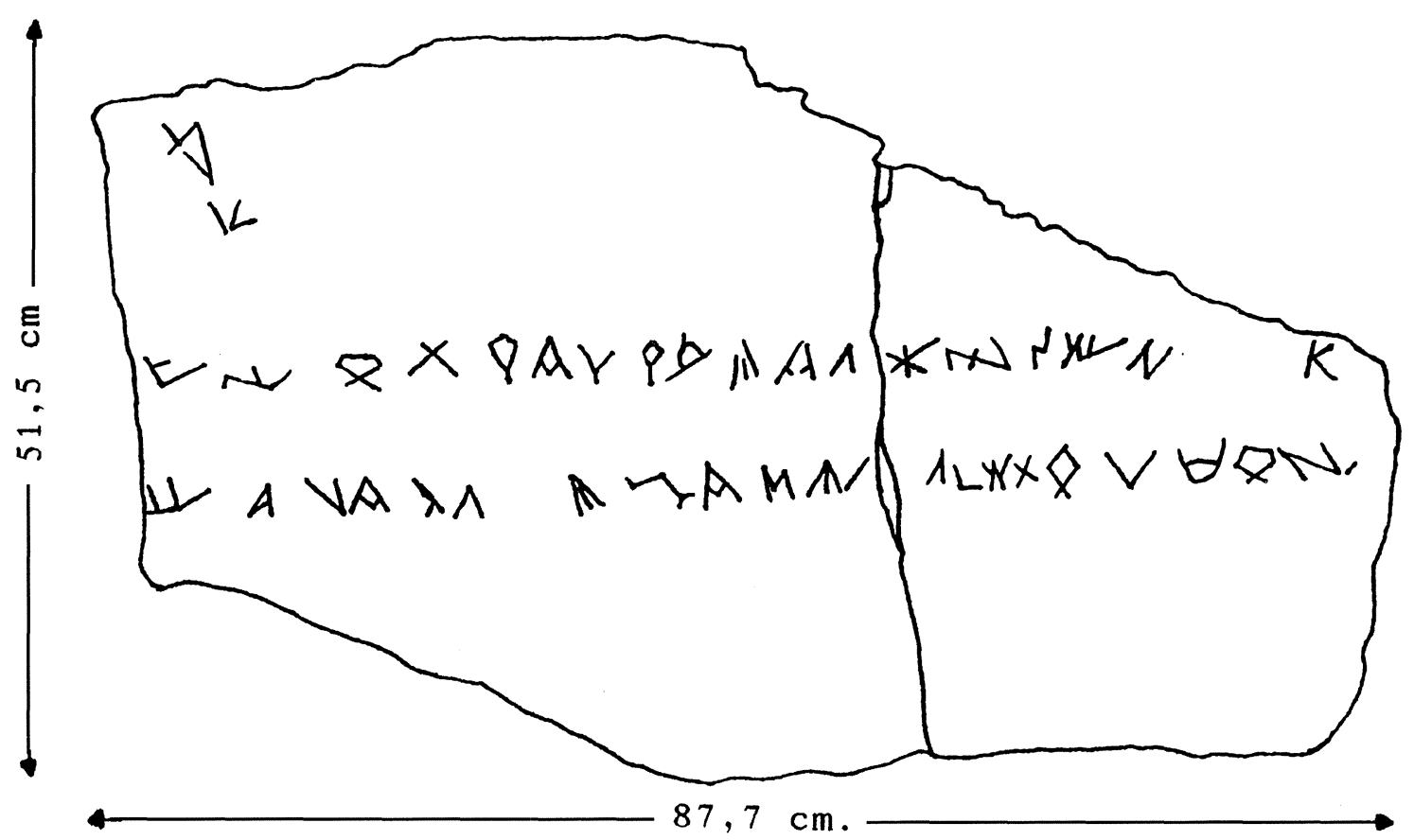

Fig. 2.-Dibujo de la inscripción de Los Maíllos (signos más seguros).

crituras paleohispánicas, ya que hasta el momento los hallazgos más occidentales de la misma en la mitad sur de la península ibérica se habían producido en la provincia de Valencia, con la única excepción de la inscripción aparecida en El Alcornocal (CO). Pero ésta no garantiza el uso efectivo de la misma en la zona, dado que se trata de un epígrafe sobre un cuenco de plata y, por tanto, fácilmente transportable. En nuestro caso, en cambio, la posibilidad de que la inscripción haya sido encontrada lejos de su emplazamiento original parece descartable, ya que el soporte son unos riscos de gran peso. Así pues, el hallazgo de Los Maíllos supone el primer testimonio de las escrituras paleohispánicas en esta parte del territorio castellano-manchego e implica el conocimiento de al menos una variedad de las mismas en una zona del interior de la Península en la que hasta el momento no había indicios de su utilización. De hecho, los lugares más cercanos a Belvís de la Jara donde hasta el momento habían aparecido epígrafes en signarios paleohispánicos son Siruela (Badajoz) y Madroñera (Cáceres), pero los hallazgos no son directamente relacionables, ya que en estos dos casos se trata de inscripciones en escritura del suroeste ${ }^{3}$ y no en el signario ibérico clásico.

${ }^{3}$ Para un catálogo de las inscripciones conocidas en escritura del suroeste vid. Correa (1993: 536-538).
La inscripción presenta un cierto cuidado: el campo epigráfico ha sido preparado para recibirla, ofreciendo una superficie plana sobre la cual se ha grabado la inscripción. Las propias vetas de la piedra han sido aprovechadas como pauta para su realización.

Antes de pasar a la transcripción de la misma hemos de hacer otra observación general. La inscripción ha estado visible durante mucho tiempo en la pared en la que se halló. Aunque obviamente las gentes del lugar no debían de reconocer que en ella había algún tipo de escritura, sí era perceptible que había líneas incisas. Esto ha podido motivar que en tiempos recientes se hayan grabado otras líneas que dificultan enormemente la tarea de discernir qué signos están realmente presentes en la inscripción, por lo que la transcripción que ofrecemos ha de tomarse con ciertas reservas.

La inscripción consta como mínimo de dos líneas de escritura, que son las que analizaremos a continuación. Además, en la zona superior izquierda aparecen al menos dos signos sueltos (fig. 2) y cabe, incluso, la posibilidad de que existieran líneas inscritas en caracteres más pequeños por encima y por debajo de las dos que mejor se perciben (fig. 3). Sin embargo, no resulta posible intentar una transcripción, pues no logramos identificar signos coherentes. 


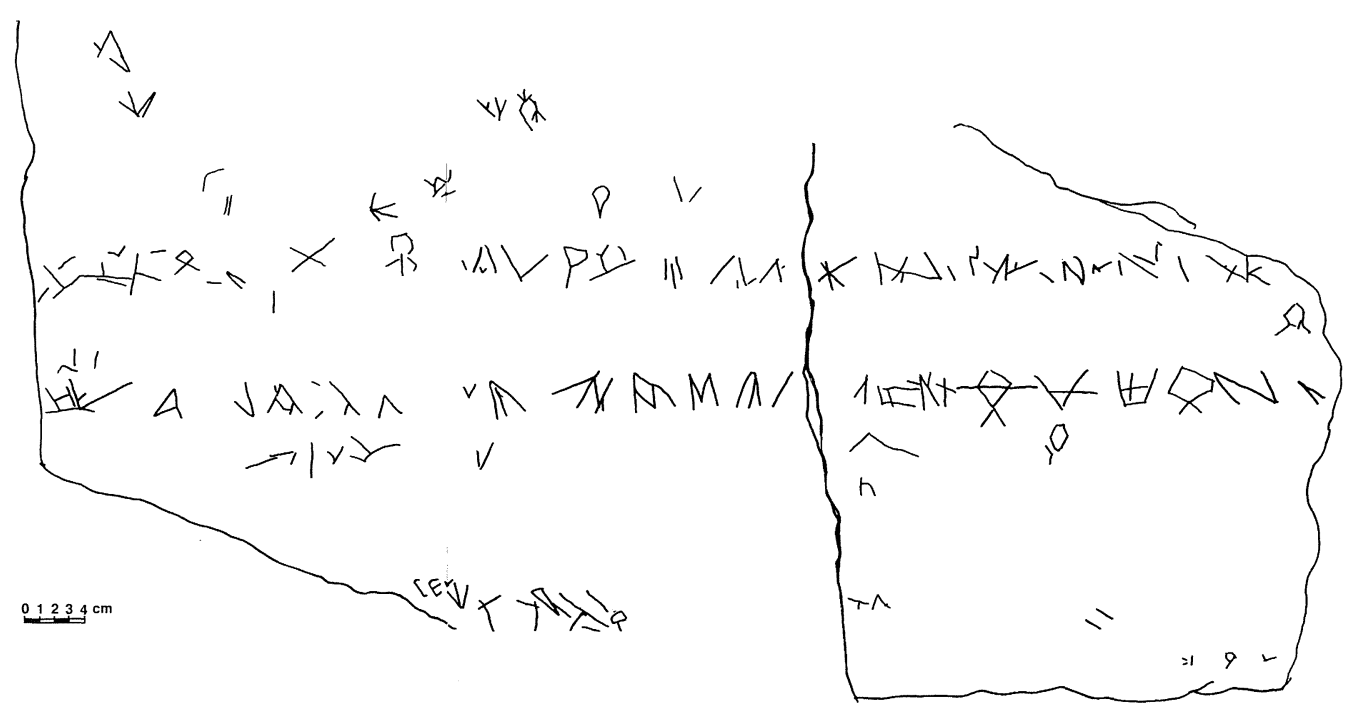

Fig. 3.-Calco de la inscripción de Los Maíllos.

\subsection{Transcripción}

La identificación de los signos que aparecen en las dos líneas seguras de escritura puede verse en la fig. 2. La transcripción que proponemos a partir de dicha identificación es la siguiente ${ }^{4}$ :

- lín. 1:

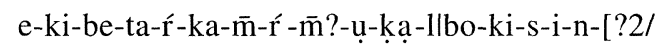
3]-ke

- lín. 2:

to?-r- $\bar{m}-k a-?-1-[? 1]-u-?-k a-s-u-? \mid 1-?-i ?-t a-b e-\bar{m}-$ ?-be-ki

\subsection{Observaciones paleográficas}

La inscripción, como decíamos, ha sido realizada en semisilabario levantino. La lectura ha de realizarse de izquierda a derecha según muestra la orientación de los signos dentro de la propia inscripción.

En la inscripción sólo se documentan los siguientes signos:

$\begin{array}{cccc} & \mathrm{e} & \mathrm{i} & \\ \mathrm{ka} & \mathrm{ke} & \mathrm{ki} & \\ \text { ta } & & & \begin{array}{c}\text { to } \\ \text { bo }\end{array} \\ & \text { be } & & \\ \mathrm{s} & \mathrm{r} & \mathrm{n} & 1 \\ \mathrm{~s} & \mathrm{r} & \overline{\mathrm{m}} & \end{array}$

${ }^{4}$ Por I notamos el punto por donde se encuentra fracturada la inscripción.
Como rasgo significativo hemos de llamar la atención sobre la utilización del signo $\mathrm{Y}=\overline{\mathrm{m}}$, lo que parece apuntar a una cronología no temprana de la inscripción ${ }^{5}$.

Siguiendo la clasificación paleográfica que de los signos de esta escritura hace Untermann (1990: 246-247), los que aparecen en esta inscripción son los siguientes: e1, u2, ka3, ki7, be1, bo3, ś2, r 3, $\overline{\mathrm{m}} 1, \mathrm{n} 2,11$. Por lo que a i, to, ke, s, r y una de las variantes de ki se refiere, los signos empleados la inscripción no se corresponden exactamente con ninguno de los tipos que establece Untermann.

Dejando de lado los signos de uso general dentro de este sistema de escritura, como es el caso de e1, r 3, 11 y n2, y que, por tanto, no son significativos para establecer comparaciones, la mayor parte de los otros se encuentra documentada en Ampurias y Sagunto, lo que sólo en parte se explica por el hecho de que el volumen de epigrafía de estos dos centros es amplio, pues no hay coincidencias significativas con otros centros bien representados epigráficamente, como Azaila. No llegamos a discernir, sin embargo, la interpretación que se puede dar a este hecho.

\subsection{La lengua de la inscripción}

El lugar donde ha aparecido la inscripción era territorio vetón en la antigüedad ${ }^{6}$, pues parece que

\footnotetext{
5 Vid. Untermann (1990: 137).

6 Para la delimitación del territorio vetón vid. Roldán (1968/69: 101-106), Salinas de Frías (1986: 21-22) y Sayas y López Melero (1991: 79-80). Para los límites concretos en la provincia de Toledo puede seguirse el trabajo de González-Conde (1986), aunque actualmente el área de dispersión
} 
hay que asumir que este pueblo se extendía por el occidente de la actual provincia de Toledo hasta Talavera de la Reina, que generalmente se identifica con la antigua Caesarobriga. Así pues, en consonancia con lo que sabemos de los vetones, cuya lengua debía de ser indoeuropea según lo que podemos deducir a partir de su onomástica ${ }^{7}$, se esperaría que la lengua de una inscripción indígena aparecida en su territorio nos mostrara una lengua de dicha familia lingüística. Sin embargo, de acuerdo con la transcripción que hemos realizado no resulta posible analizar la lengua de la inscripción como indoeuropea, sino que la interpretación más verosímil apunta hacia el ibérico.

Aunque con todas las cautelas, puede proponerse el siguiente análisis. En la primera línea, tras tres signos previos, se documenta la secuencia -taŕ, que Untermann (1990: 233) analiza como uno de los elementos utilizados en la antroponimia ibérica. De ello podría deducirse tal vez que la secuencia kibe que la precede constituye el primer elemento del nombre propio, que podría ser entonces una variante de los elementos kibas $/ k_{i b a}{ }^{8}$. Por lo que hace al $k a$ que sigue, siguiendo en la misma línea de interpretación, podría tratarse del elemento $k a$ que Untermann (1987: 36-38) aísla en sintagmas que incluyen nombres de personas y para el que postula que se trata de una marca de ergativo. En cambio, ya desde el punto de vista fonético, resulta extraña la combinación $-\bar{m} \bar{r}_{\bar{m}}$ - que sigue en la inscripción.

En cuanto a $u k a l$, si es que ésa es la lectura correcta, es posible que la misma secuencia se encuentre atestiguada en un grafito de Ampurias (Untermann, 1990: C.1.9) y quizá sea identificable también con el inicio de ukalkebars en el plomo de Yátova (Untermann, 1990: F.20.1). Por otro lado, y dado que -in, que aparece tras bokis detrás de la fractura, tal vez pueda interpretarse como un sufijo de nombre propio femenino ${ }^{9}$, podemos sugerir que la secuencia ukalbokis sea un nombre femenino.

de los verracos, que es uno de los criterios utilizados por esta investigadora, ha variado ligeramente; vid. los nuevos hallazgos en Alvarez-Sanchís (1993: 160-162). Por otra parte, la difusión del culto de Ataecina, de la que se conocen varios epígrafes en el occidente toledano, también puede utilizarse como un criterio de delimitación, siguiendo las observaciones de Abascal (1995: 103-105).

7 Vid. Albertos (1983: 869-872).

${ }^{8}$ Sobre los cuales vid. Untermann (1990: 226) y Velaza (1991: 91-92).

9 Vid. Untermann (1990: 204-205). En realidad la existencia de un sufijo -in como tal puede basarse únicamente en aiunin frente al primer elemento de aiunesker y aiunibaiser, pues en los demás casos en que aparece lo hace dentro de la combinación -unin-, que puede ser un elemento léxico con el significado de «mujer» o «hija», posibilidad que contempla Untermann (1990: 205) siguiendo a Schmoll.
Para el elemento bokis sólo podemos ofrecer como posible paralelo la secuencia boka que se atestigua repetidamente en grafitos cerámicos de Azaila (Untermann 1990: E.1.117-120, 289 y 379). Para las secuencias que se atestiguan en la segunda línea no hemos conseguido encontrar paralelos que puedan ser significativos.

\subsection{Ensayo de interpretación}

Habida cuenta de la posible presencia de dos antropónimos, uno masculino y uno femenino, en la primera línea del epígrafe, nos sentimos inclinados a pensar en una inscripción de carácter funerario, carácter con el que no creemos que esté en contradicción el soporte material, aunque para él carecemos de paralelos dentro de la epigrafía indígena de la península ibérica.

Por otra parte, si el elemento $k a$ fuera realmente una marca de ergativo, se podría apuntar hacia una interpretación de la relación entre los dos personajes que aparecen en la inscripción en el sentido de que el hombre realizó la sepultura para la mujer, sin que podamos precisar más dado nuestro desconocimiento casi total de la gramática y el léxico ibéricos.

\section{CONTEXTUALIZACIÓN ARQUEOLÓGICA}

Jiménez de Gregorio ${ }^{10}$ ha llevado a cabo varias inspecciones de la zona en la que apareció la inscripción sin que pudiera hallar ningún elemento arqueológico relacionable con el epígrafe. Posteriormente nosotros mismos, en compañía del Prof. Juan Pereira, el Dr. Arturo Ruiz y D. ${ }^{a}$ Alicia Torija procedimos a realizar un nuevo reconocimiento del terreno sin que obtuviéramos ningún resultado. Sin embargo, hay que llamar la atención sobre el hecho de que, a pesar de la poca atención que, en general, se viene prestando a la investigación sobre los elementos ibéricos en la provincia de Toledo, el hallazgo tal vez no sea un unicum en la comarca, pues a pocos kilómetros del lugar del lugar donde apareció la inscripción, en la ermita de la Virgen de Barbarroya (término municipal de Aldeanueva de Barbarroya) - un lugar habitado en época hispanorromana según muestran los hallazgos de cerámica de esa época (Jiménez de Gregorio 1958: 200)- se encontraba incrustada en el arco del crucero una figura que, según la descripción de Jiménez de Gregorio

${ }^{10}$ Comunicación personal. 
(1958: 199-200; 1992: 9), constaba de cabeza de león con rasgos humanos y garras de animal y que él sugirió interpretar como una esfinge ibérica.

Aun con las salvedades pertinentes en cuanto al uso del término «ibérico» en la bibliografía antigua, se puede hacer referencia a otros hallazgos de la zona. Así, Jiménez de Gregorio (1953: 27) da cuenta de la aparición en Cascajoso (término de Belvís) de un resto de construcción circular de mampostería con cantos rodados de $0,60 \mathrm{~m}$ de anchura, 0,40 $\mathrm{m}$ de altura y $7,50 \mathrm{~m}$ de radio en cuyo interior se encontró una vasija de barro de ejecución tosca, con ancha base y sin bordes, de una anchura máxima de $15 \mathrm{~cm}$ y altura de $20 \mathrm{~cm}$, que contenía pequeños huesos y cenizas y que él interpretó como ibérica. Jiménez de Gregorio (1992: 11) también alude a unos restos «ibéricos» aparecidos en la labranza de El Viñazo (Belvís), consistentes ${ }^{11}$ en un fondo de cabaña, además de información de hallazgos de vasijas por parte de los lugareños.

Por otra parte, en la labranza de Los Villarejos (término municipal de Alcaudete de la Jara), apareció (Jiménez de Gregorio 1950: 113-114 y 1992: 8) una necrópolis que constaba al menos de ocho hileras de enterramientos paralelos y concéntricos, con una laja de piedra clavada en el suelo marcando uno de los laterales de cada fosa. Al parecer, cada fosa contenía una vasija, sin labra y de realización tosca. Los dos ejemplares que describe Jiménez de Gregorio (1950: 113-114), tienen, respectivamente, las siguientes dimensiones: el primero, $75 \mathrm{~cm}$ de alto, $62 \mathrm{~cm}$ de boca, $42 \mathrm{~cm}$ de base y $13,2 \mathrm{~cm}$ de ancho y el segundo, $77 \mathrm{~cm}$ de alto, $68 \mathrm{~cm}$ de boca, $49 \mathrm{~cm}$ de base y $14,5 \mathrm{~cm}$ de ancho. Pero a pesar de ser de dimensiones parecidas, los dos ejemplares se diferencian en que el primero cuenta con un pie muy marcado mientras que el labio apenas está esbozado; el segundo, en cambio, carece de pie definido $\mathrm{y}$, por contra, tiene el labio bien marcado ${ }^{12}$. Jiménez de Gregorio (1950: 114) sugiere —aunque con reservas- su interpretación como materiales ibéricos.

El cuadro general en el que en principio cabría insertar estos materiales, cuya cronología es difícil de afinar dadas las circunstancias de su hallazgo, es el del proceso de iberización que en la época del Hierro II afecta a toda la Meseta Sur (Blasco, 1992: 292295) y que, aunque es más temprano al sur del Guadiana, en la zona que nos interesa no ha podido alcanzar su culminación antes del siglo Iv. El proceso de iberización de esta zona se entiende general-

\footnotetext{
"Comunicación personal del Prof. Jiménez de Gregorio.
}

12 Vid. la fotografía en Jiménez de Gregorio (1950: fig. 8). mente como debido a influjos procedentes del área extremeña (Almagro-Gorbea, 1988: 172), lo que cuadra bien con el hecho de que en los periodos anteriores del Bronce Final y del Hierro I los hallazgos de la zona, como el ajuar funerario de Las Fraguas (en la Dehesa de Manzanas, término de Las Herencias), la tumba de la Casa del Carpio (Belvís de la Jara) y la estela aparecida en el lugar de Los Castillos (término de Las Herencias), muestran la existencia de «tradiciones indígenas asociadas a influencias procedentes del suroeste peninsular que ponen en contacto a este territorio con Extremadura y, a su través, con el área tartéssica y fenicia más occidental» (Fernández Miranda y Pereira, 1992: 71).

Así pues, la inscripción de Los Maíllos plantea un interesante problema de carácter histórico-arqueológico, puesto que, como hemos visto, por sus características apunta a influjos procedentes del área levantina, mientras que, según la interpretación generalmente aceptada, las corrientes culturales dominantes en la zona, tanto en la época del Hierro II como del Hierro I, proceden del área extremeña.

No podemos finalizar sin hacer referencia al yacimiento de arroyo Manzanas (Las Herencias), cuya cronología (Moreno Arrastio, 1990: 291-292) abarca desde la época de Cogotas I hasta finales del siglo II a.C./principios del i a.C., por lo que algunos de sus niveles han de ser contemporáneos de al menos algunos de los hallazgos que mencionábamos más arriba. En los niveles inferiores de este yacimiento (Moreno Arrastio, 1990: 283-284) aparecen materiales que pueden asociarse a los de la tumba del Carpio y, además, se ha hallado en él otra estela de guerrero de las conocidas como «estelas extremeñas» (Moreno Arrastio, 1995). Sin embargo, para los niveles superiores previos al abandono del poblado en el siglo i a.C., que son los que más directamente nos interesan, no parece que se pueda hablar de una iberización de la cultura material.

\section{BIBLIOGRAFÍA}

Abascal Palazón, J. M., 1995: Las inscripciones latinas de Santa Lucía del Trampal (Alcuéscar, Cáceres) y el culto de Ataecina en Hispania, AEspA 68, 31-105.

Albertos, M. ${ }^{a}$ L., 1983: Onomastique personnelle indigène de la Péninsule Ibérique sous la domination romaine, $A N R W$ II 29, 2, 853-892.

Almagro-Gorbea, M., 1988: Las culturas de la Edad del Bronce y de la Edad del Hierro en Castilla-La Mancha, en: Actas del Primer Congreso de Historia de Castilla-La Mancha, vol. 2: Pue- 
blos y culturas prehistóricus y protohistóricas, Talavera de la Reina, 163-180.

Álvarez-SANChís, J. R., 1993: En busca del verraco perdido (aportaciones a la escultura zoomorfa de la Edad del Hierro en la Meseta), Complutum 4, 157-168.

Blasco Bosqued, M. ${ }^{a}$ C., 1992: Etnogénesis de la Meseta Sur, en: Martín Almagro-Gorbea y Gonzalo Ruiz Zapatero (eds.), Paleoetnología de la Península Ibérica (=Complutum 2-3), Madrid, 281-297.

Correa, J. A., 1993: El signario de Espanca (Castro Verde) y la escritura tartesia, en: FrANCISCO VILlAR y Jürgen UNTERMANN (eds.), Lengua $y$ cultura en la Hispania prerromana (Actas del $\mathrm{V}$ Coloquio sobre Lenguas y Culturas Prerromanas de la Península Ibérica), Salamanca, 521562.

Fernández Miranda, M. y J. Pereira, 1992: Indigenismo y orientalización en la tierra de Talavera, en: Actas de las Primeras Jornadas de Arqueología de Talavera de la Reina y sus Tierras, Toledo, 57-94.

González-Conde Puente, M. ${ }^{a}$ P., 1986: Elementos para una delimitación entre vettones y carpetanos en la provincia de Toledo, Lucentum 5, 8793.

JiMÉNEZ DE GREGORIo, F., 1950: Hallazgos arqueológicos en La Jara II, AEspA 23, 105-117.

—, 1953: Historia de Belvís, Madrid.

—, 1958: Hallazgos arqueológicos en La Jara VIII, AEspA 31, 199-204.
—, 1989: Hallazgos arqueológicos en la provincia de Toledo (VI), Anales Toledanos 26, 7-59.

-, 1992: Aproximación al mapa arqueológico del occidente provincial toledano, en: Actas de las Primeras Jornadas de Arqueología de Talavera de la Reina y sus Tierras, Toledo, 5-38.

Moreno Arrastio, F. J., 1990: Notas al contexto de arroyo Manzanas (Las Herencias, Toledo), en: Actas del Primer Congreso de Arqueología de la Provincia de Toledo, Talavera de la Reina, 275308.

- 1995: La estela de Arroyo Manzanas (Las Herencias II, Toledo), Gerión 13, 275-294.

Roldán Hervás, J. M., 1968/69: Fuentes antiguas para el estudio de los Vettones, Zephyrus 19/20, 73-106.

SALINAS DE FRÍAs, M., 1986: La organización tribal de los vettones, Salamanca.

Sayas Abengoechea, J. J. y R. López Melero, 1991: Vettones, en: José María Solana Sainz (ed.), Las entidades étnicas de la Meseta Norte de Hispania en época prerromana (Anejos de Hispania Antiqua), Valladolid, 74-123.

Untermann, J., 1987: La gramática de los plomos ibéricos, en: Joaquín Gorrochategui, José Luis Melena y Juan Santos (eds.), Actas del IV Coloquio sobre Lenguas y Culturas Paleohispánicas (=Veleia 2-3), Vitoria, 35-56.

-, 1990: Monumenta Linguarum Hispanicarum, vol. III, 2 tomos, Wiesbaden.

VelazA, J., 1991: Léxico de inscripciones ibéricas (1976-1989), Barcelona. 